\title{
Virus-like Replicon Particle Co-expression of PRRSV GP5 and $M$ Proteins
}

\begin{abstract}
A.S. Leaflet R2175
Matthew M. Erdman, postdoctoral associate; D.L. (Hank) Harris, professor of animal science; Kurt I. Kamrud, AlphaVax, Inc

\section{Summary and Implications}

VRP were constructed that co-express porcine reproductive and respiratory syndrome virus (PRRSV) GP5 and $\mathrm{M}$ proteins and form a heterodimer. This co-expressing VRP is an attractive candidate for a novel PRRSV vaccine based on previous work with equine arteritis virus (EAV).

\section{Introduction}

Virus-like replicon particles (VRP) derived from the alphavirus Venezuelan equine encephalitis (VEE) is a single cycle vector not capable of propagating past the initial cell infected. VRP have been previously used to show that coexpression of the $G_{L}$ and $M$ proteins of EAV are required for protection. We have recently developed VRP coexpressing GP5 and M proteins of PRRSV, however there are no previous reports of immunizing swine with VRP vaccines. The purpose of this study was to determine the potential for using VRP vaccines in pigs.
\end{abstract}

\section{Materials and Methods}

VRPs were constructed as expressing PRRSV proteins. The PRRSV ORF5 and ORF6 genes were cloned into replicon vectors individually. Replicons that produced the correct sized subgenomic transcripts and at the highest level were chosen to generate double subgenomic replicons coexpressing both (Figure 1). Replicons were analyzed by IFA, Western blot, and northern blot. Each replicon was then packaged into a VRP by supplying alphavirus structural proteins in trans. VRP were incubated for 18 hours on Vero cells followed by cell lysis and Western blot analysis.

\section{Results and Discussion}

Analysis of replicons indicated that constructs were producing the correct sized subgenomic transcripts and correct PRRSV proteins detected by PRRSV convalescent pig serum. Western blot analysis of Vero cell lysates indicated that the VRP co-expressed the GP5 and M monomers as well as the GP5-M heterodimer (Figure 2).

VRP can co-express PRRSV GP5 and M proteins in heterodimer form. This work supports the in vivo evaluation of GP5-M VRP as a novel vaccine for PRRSV. Vaccinationchallenge trials in pigs are in progress.
Figure 1. Design of replicon co-expressing PRRSV GP5 and $M$ proteins.

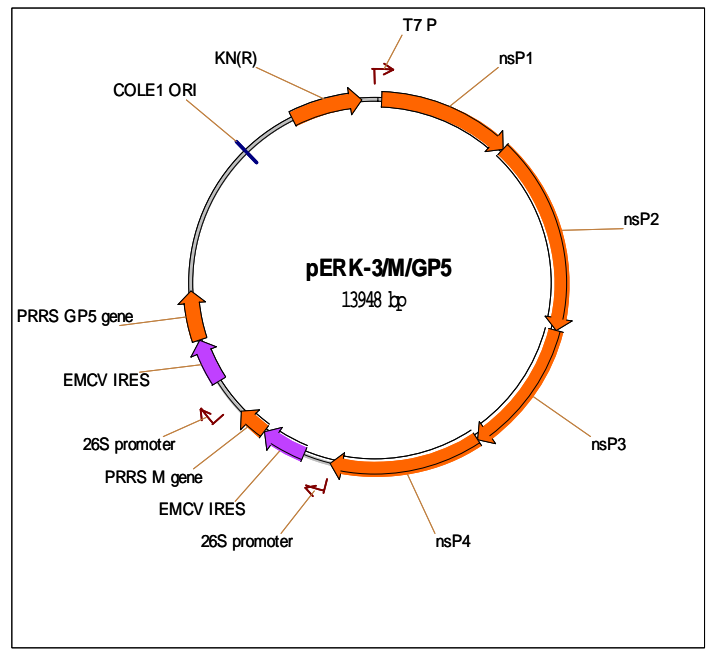

Figure 2. Non-reduced western blot. Lane 1: ladder, Lane 2: co-expressed GP-M, Lane 3: neg control



Acknowledgments

This work was supported by the USDA PRRS CAP. 